\title{
Admitting lung cancer patients to intensive care for organ dysfunction: a complex decision process
}

\author{
Anne-Pascale Meert, Thierry Berghmans and Jean-Paul Sculier
}

Affiliation: Service des Soins Intensifs et Urgences Oncologiques \& Oncologie Thoracique, Institut Jules Bordet, Université Libre de Bruxelles, Brussels, Belgium.

Correspondence: A-P. Meert, Service des Soins Intensifs et Urgences Oncologiques \& Oncologie Thoracique, 1, rue Héger Bordet, Institut Jules Bordet, B-1000 Bruxelles, Belgium. E-mail: ap.meertabordet.be

@ERSpublications

With adequate selection, intensive care and mechanical ventilation should not be considered futile in lung cancer http://ow.ly/E3lB1

Physicians in charge of patients with lung cancer not only face the cancer itself but also major comorbidities due to tobacco exposure. In addition to complications of lung cancer and anticancer treatments, chronic obstructive pulmonary disease exacerbations and cardiovascular disease, for example, are situations that can potentially lead patients with lung cancer to emergency wards and, finally, intensive care units (ICUs). Lung cancer represents up to $15-20 \%$ of ICU admissions in cancer patients. The reasons for ICU admission are primarily respiratory problems, with pneumonia being the leading cause, and sepsis and shock as the second and third aetiologies.

During recent decades, the poor prognosis of cancer patients, both with haematological and with solid tumours, has prompted the decision for the ICU to refuse admission to these patients. Much progress in critical care has moved this common attitude from a nihilistic approach to a complex process for selecting patients before referring cancer patients to the ICU. It has been clearly demonstrated that the prognosis of cancer patients during their ICU stay is mainly dependent upon acute physiological disturbances induced by the complication that led the patient to the ICU $[1,2]$. However, after recovery, the prognosis is again determined by the characteristics of the underlying oncological disease. These conclusions are of value in unselected oncology populations [2] and in specific groups of cancer patients requiring invasive mechanical ventilation[3], noninvasive ventilation [4] or renal replacement therapy $[1,5]$.

Along with the overall improvement in the prognosis of ICU cancer patients, the same trend was observed for lung cancer [5], with hospital mortality ranging from $24 \%$ to $65 \%[6,7]$. It is also important to consider that the prognosis of lung cancer patients surviving to the ICU stay is relatively poor, with a 6-month mortality rate of $73 \%$, and only two-thirds of those receiving further anticancer treatment survive [8]. In this issue of the European Respiratory Journal, the article by TOFFART et al. [9] reflects this situation, with hospital mortality of $\sim 60 \%$ for patients admitted to ICU and a 1-year survival after discharge from the ICU of $12 \%$. Using a population similar to common descriptions, the authors asked how the decisions for referral of lung cancer patients to ICU were taken.

Decision for ICU admission is certainly a complex process in which multiple stakeholders (medical oncologists, pneumologists, intensivists, general practitioners, patients and their relatives) are involved. They have to consider objective (cancer stage and status, general conditions and comorbidities, etc.) but also subjective constraints of a philosophical, psychological or religious nature in their decision. ToffarT et al. [9] reported on 140 consecutive lung cancer patients with at least one organ dysfunction admitted to a single general hospital during a 23-month period. What is the main information provided by that

Received: Oct 272014 | Accepted: Oct 312014

Conflict of interest: None declared.

Copyright OERS 2015 
prospective pilot study? The authors underline the prominent role of the (pneumo)oncologists and the patient in the decision for ICU referral. The first reason for not referring patients was refusal by the patient or their family. By showing the major importance of the patients/family in refusing ICU admission, this study reinforces the fact that anticancer treatment, which includes intensive care, must be centred on patients' wishes and expectations. Lung cancer prognosis is another major consideration in the decision process before admitting lung cancer patients into ICU. To approach this major point, the physician needs to have an adequate knowledge of lung cancer behaviour, prognosis and treatment but also to offer a therapeutic project at hospital discharge. This is the pivotal role of the (pneumo)oncologists who, in the study by TOFFART et al. [9], were involved in $86 \%$ of the decisions. Intensivists only regain importance when considering the final decision for ICU admission.

The present study has the major advantage of showing us an unbiased view of an unselected lung cancer population treated in a general hospital. Among the three independent factors involved in the decision for ICU referral, the patient's decision and the absence of disease progression are of major interest. As suggested by the authors, further multicentric studies could be of value for confirming the present data, avoiding potential biases due to the unicentric nature of the present one. Incorporating cancer centres with dedicated oncological ICUs could also add value for developing a useful guide for (pneumo)oncologists and intensivists in this sensitive situation.

The decision to permit or refuse ICU admission for a patient with lung cancer is a complex process that has to involve close collaboration between health stakeholders (pneumologists, oncologists and intensivists), and the patient and their relatives. The final decision must be centred on the patient's expectations. It will integrate not only objective parameters focusing on cancer prognosis and therapeutic planning but also subjective variables. People in charge of ICUs have to consider that the prognosis of lung cancer patients has been changed during the last decade not only by better knowledge of the tumoural biology and the development of targeted therapies but also by improved resuscitation techniques. Currently, intensive care and mechanical ventilation should not be considered futile care in this population provided an adequate selection of the patients is performed.

\section{References}

1 Berghmans T, Meert AP, Markiewicz E, et al. Continuous venovenous haemofiltration in cancer patients with renal failure: a single-centre experience. Support Care Cancer 2004; 12: 306-311.

2 Sculier JP, Paesmans M, Markiewicz E, et al. Scoring systems in cancer patients admitted for an acute complication in a medical intensive care unit. Crit Care Med 2000; 28: 2786-2792.

3 Vallot F, Paesmans M, Berghmans T, et al. Leucopenia is an independent predictor in cancer patients requiring invasive mechanical ventilation: a prognostic factor analysis in a series of 168 patients. Support Care Cancer 2003; 11: $236-241$.

4 Meert AP, Berghmans T, Hardy M, et al. Non-invasive ventilation for cancer patients with life-support techniques limitation. Support Care Cancer 2006; 14: 167-171.

5 Wallace S, Ewer MS, Price KJ, et al. Outcome and cost implications of cardiopulmonary resuscitation in the medical intensive care unit of a comprehensive cancer center. Support Care Cancer 2002; 10: 425-429.

6 Andréjak C, Terzi N, Thielen S, et al. Admission of advanced lung cancer patients to intensive care unit: a retrospective study of 76 patients. BMC Cancer 2011;11: 159 .

7 Slatore CG, Cecere LM, Letourneau JL, et al. Intensive care unit outcomes among patients with lung cancer in the surveillance, epidemiology, and end results - Medicare registry. J Clin Oncol 2012; 30: 1686-1691.

8 Roques S, Parrot A, Lavole A, et al. Six-month prognosis of patients with lung cancer admitted to the intensive care unit. Intensive Care Med 2009; 35: 2044-2050.

9 Toffart A-C, Pizarro CA, Schwebel C, et al. Selective criteria for intensive care unit referral of lung cancer patients: a pilot study. Eur Respir J 2015; 45: 491-500. 